\title{
Joint Application of Bilinear Operator and F-Expansion Method for $(2+1)$-Dimensional Kadomtsev-Petviashvili Equation
}

\author{
Shaolin Li, Yinghui He, and Yao Long \\ Department of Mathematics, Honghe University, Mengzi, Yunnan 661100, China \\ Correspondence should be addressed to Shaolin Li; shaolinli@163.com and Yao Long; yaolong04@163.com
}

Received 5 August 2014; Accepted 2 November 2014; Published 12 November 2014

Academic Editor: Marcelo M. Cavalcanti

Copyright (C) 2014 Shaolin Li et al. This is an open access article distributed under the Creative Commons Attribution License, which permits unrestricted use, distribution, and reproduction in any medium, provided the original work is properly cited.

\begin{abstract}
The bilinear operator and F-expansion method are applied jointly to study $(2+1)$-dimensional Kadomtsev-Petviashvili (KP) equation. An exact cusped solitary wave solution is obtained by using the extended single-soliton test function and its mechanical feature which blows up periodically in finite time for cusped solitary wave is investigated. By constructing the extended doublesoliton test function, a new type of exact traveling wave solution describing the assimilation of solitary wave and periodic traveling wave is also presented. Our results validate the effectiveness for joint application of the bilinear operator and F-expansion method.
\end{abstract}

\section{Introduction}

In the past few decades, much effort has been devoted to the investigation of dynamical behaviours of nonlinear evolution equation. Traveling wave, one of the spatial dynamics analyses, always plays a significant role and attracts more and more of the experts' and scholars' attention. There has been much literature on traveling wave of nonlinear evolution equation due to the abundant type of nonlinear traveling wave and some well-known concepts (e.g., solitary wave [1-3], periodic wave $[4,5]$, kink wave [6], cusped wave [7], etc.) have been used and generalized extensively. To understand the inherent essence and evolution mechanism of these nonlinear traveling waves, seeking the exact traveling wave solutions has been recognized. In recent years, much efforts have been spent on this task and many significant methods have been established such as variational iteration method [8], homotopy perturbation method $[9,10]$, Fan subequation method $[11,12]$, exp-function method [13], Hirota's bilinear method $[14,15], G^{\prime} / G$-expansion method [16, 17], and F-expansion method [18-21]. In most of the existing literature, authors always study the improvement of the adopted method to obtain more forms of solutions. However, to the best of our knowledge, how to realize the joint applications of different methods is still challenging and open work. In this paper, we choose the classical nonlinear evolution equation, $(2+1)$-dimensional Kadomtsev-Petviashvili (KP) equation, as an example to validate the effectiveness of the proposed method.

The $(2+1)$-dimensional KP equation [14] is written as

$$
u_{x t}-u_{x x x x}-3\left(u^{2}\right)_{x x}=3 p^{2} u_{y y}
$$

where $u: R_{x} \times R_{y} \times R_{t} \rightarrow R$ and $p^{2}= \pm 1$ measure the positive and negative transverse dispersion effects. Equation (1) with $p^{2}=1$ and $p^{2}=-1$ are called KP-I equation and KP-II equation, respectively. In recent years, kinds of research fields and solution types of KP equation have been studied extensively in various aspects [22-24]; exact multiple solitary wave solution, periodic solitary wave solution, quasiperiodic solutions, and so forth have been obtained. In the past works, the resonance interaction phenomenon between periodic solitary wave and line soliton was investigated and spatial-temporal bifurcation and deflexion of solitary wave were exhibited [14, 15].

The rest of the paper is organized as follows. In Section 2, we combine the bilinear operator with F-expansion method to solve KP equation. By single-soliton test approach, a new type of solitary wave solution which possesses cusped structure is obtained. In Section 3, an exact expression describing the interaction of solitary wave and periodic traveling wave 
is obtained by the extending of double-soliton test approach. Conclusions are drawn in Section 4.

\section{Cusped Solitary Wave Solution}

Introduce an independent transformation

$$
u(x, y, t)=2(\ln F)_{x x},
$$

where $F=F(x, y, t)$ is an unknown real function. Substituting (2) into (1), we can obtain the bilinear form of KP equation:

$$
\left(D_{x} D_{t}-D_{x}^{4}-D_{x}^{2}-p^{2} D_{y}^{2}\right) F \cdot F=0,
$$

where Hirota's bilinear operator “ $D$ ” is defined by

$$
\begin{aligned}
D_{x}^{k} D_{y}^{m} D_{t}^{n} f(x, y, t) \cdot g(x, y, t) \\
=\frac{\partial^{k}}{\partial s^{k}} \frac{\partial^{m}}{\partial \sigma^{m}} \frac{\partial^{n}}{\partial \tau^{n}} \\
\quad \times\left.(f(x+s, y+\sigma, t+\tau) g(x-s, y-\sigma, t-\tau))\right|_{s=0, \sigma=0, \tau=0} .
\end{aligned}
$$

Consider the traveling wave transformation

$$
F(x, y, t)=F(\xi), \quad \xi=k x+l y+\omega t+\gamma_{0},
$$

where $k, l$, and $\omega$ are nonzero constants and $\gamma_{0}$ is a phase constant. Equation (3) is converted to an ODE:

$$
\begin{aligned}
& \left(k^{2}+l^{2} p^{2}-k \omega\right) F^{\prime 2}-3 k^{4} F^{\prime \prime 2} \\
& \quad+4 k^{4} F^{\prime} F^{\prime \prime \prime}-F\left(\left(k^{2}+l^{2} p^{2}-k \omega\right) F^{\prime \prime}+k^{4} F^{(4)}\right)=0 .
\end{aligned}
$$

Generally, letting $F(\xi)=1+e^{\xi}$, we can obtain an exact single solitary wave solution of bilinear equation (3). In this case, we consider the extended single-soliton test function

$$
F(\xi)=\sum_{j=1}^{n} a_{j} G^{j}(\xi)
$$

where $G(\xi)$ satisfies the following auxiliary equation:

$$
\left(G^{\prime}(\xi)\right)^{2}=\sum_{k=0}^{r} b_{j} G^{j}(\xi) .
$$

The coefficients $a_{j}, b_{k}(j=0,1, \ldots, n ; k=0,1, \ldots, r)$ are undetermined constants and $n$ and $r$ are undetermined positive integers. To determine the values of $n$ and $r$, balancing the lowest order nonlinear term with the highest nonlinear terms in (6), we have a relation of $n$ and $r$ :

$$
2 n+r-2=2 n+2 r-4 .
$$

From (9), we conclude that $r=2$ and $n$ is an arbitrary positive integer. As a test, $n=2$ is taken into account; (7) and (8) are reduced into

$$
\begin{gathered}
F(\xi)=a_{0}+a_{1} G(\xi)+a_{2} G^{2}(\xi), \\
\left(G^{\prime}(\xi)\right)^{2}=h_{0}+h_{1} G(\xi)+h_{2} G^{2}(\xi),
\end{gathered}
$$

where $a_{2} \neq 0$ and $h_{2} \neq 0$.
Substituting (10) into (6), setting the coefficients of all powers of $G(\xi)$ to zero, we get a nonlinear algebraic system of coefficients $a_{0}, a_{1}, a_{2}, h_{0}, h_{1}$, and $h_{2}$ and $k, l$, and $\omega$. Solving it, we obtain

$$
\begin{gathered}
a_{0}=a_{0}, \quad a_{1}=a_{1}, \quad a_{2}=\frac{a_{1} h_{2}}{h_{1}}, \\
h_{0}=\frac{h_{1}^{2}}{4 h_{2}}, \quad h_{1}=h_{1}, \quad h_{2}=h_{2}, \\
\omega=\frac{-k^{2}-L^{2} p^{2}-4 k^{4} h_{2}}{k},
\end{gathered}
$$

where $a_{0}, a_{1}, h_{1}, k$, and $L$ are arbitrary nonzero reals and $h_{2}>$ 0 . Under the condition of $h_{0}=h_{1}^{2} / 4 h_{2}$ to solve (10), we get

$$
G(\xi)=-\frac{h_{1}}{2 h_{2}}+e^{\sigma \sqrt{h_{2}} \xi},
$$

where $\sigma= \pm 1$.

Substituting (11) and (12) into (10), by (2), we obtain an exact traveling wave solution of KP equation as follows:

$$
u(x, y, t)=\frac{32 k^{2} a_{1} h_{1} h_{2}^{3}\left(4 a_{0} h_{2}-a_{1} h_{1}\right) e^{2 \sigma \sqrt{h_{2}}\left(k x+L y-\omega t+\gamma_{0}\right)}}{\left(4 a_{0} h_{1} h_{2}-a_{1} h_{1}^{2}+4 a_{1} h_{2}^{2} e^{2 \sigma \sqrt{h_{2}}\left(k x+L y-\omega t+\gamma_{0}\right)}\right)^{2}} .
$$

A solitary wave which possesses a cusped structure is shown by (13), whose amplitude oscillates with the evolution of time. To guarantee the regularity of solitary wave, we should avoid the denominator of (13) equalling zero. So, an inequality is taken into account:

$$
\left(4 a_{0} h_{1} h_{2}-a_{1} h_{1}^{2}\right) a_{1} h_{2}^{2}>0 .
$$

For simplicity, if $a_{1}>0$, we can conclude that $a_{0}<$ $a_{1} h_{1} / 4 h_{2}$ when $h_{1}<0 ; a_{0}>a_{1} h_{1} / 4 h_{2}$ when $h_{1}>0$. Choosing a set of parameters

$$
\begin{gathered}
k=0.2, \quad L=1.2, \quad p=1, \quad \gamma_{0}=0.5, \\
a_{1}=0.2, \quad h_{1}=0.8, \quad h_{2}=0.4, \\
a_{0}=0.2, \quad \sigma=1,
\end{gathered}
$$

we exhibit a waveform of regular cusped solitary wave expressed by (13). From Figure 1, it is observed that the amplitude of cusped solitary wave periodically oscillates along the $x$-axis.

However, if $\left(4 a_{0} h_{1} h_{2}-a_{1} h_{1}^{2}\right) a_{1} h_{2}^{2}<0$, it is inevitable that the denominator of (13) equals zero for some values of $x, y$, and $t$. In other words, the equation

$$
k x+L y-\omega t+\gamma_{0}=\frac{1}{2 \sigma \sqrt{h_{2}}} \ln \left(\frac{h_{1}\left(a_{1} h_{1}-4 a_{0} h_{2}\right)}{4 a_{1} h_{2}^{2}}\right)
$$

is satisfied; the irregularity of cusped solitary wave appears. From Figures 2, 3, and 4, these singular phenomena exhibit that irregular solitary wave blows up in finite time, where we only change $a_{0}=0.2$ to $a_{0}=-0.2$ in (15). 


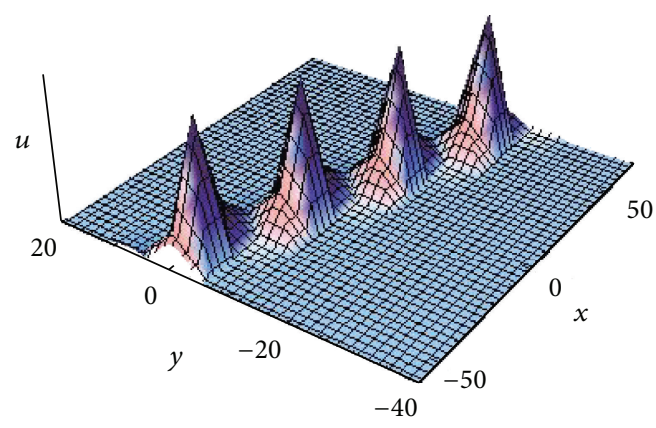

FIGURE 1: A snapshot of regular cusped solitary wave expressed by (13) at $t=1.2$, where $x \in[-60,70]$ and $y \in[-40,20]$

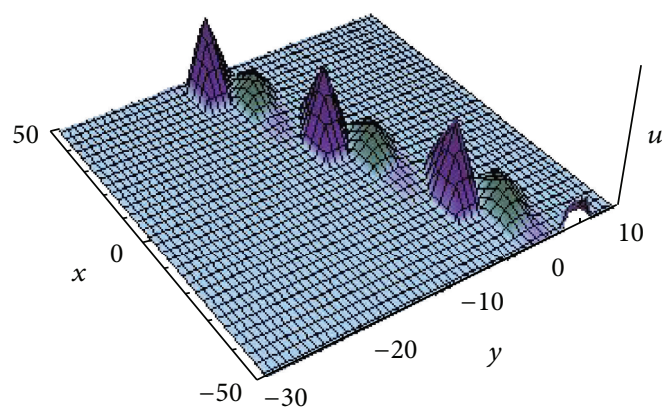

FIGURE 2: A snapshot of irregular cusped solitary wave expressed by (13) at $t=0.84$, where $x \in[-50,50]$ and $y \in[-30,10]$.

\section{Interaction of Solitary Wave and Periodic Wave}

Let

$$
F(\xi, \eta)=1+e^{\xi}+e^{\eta}+A e^{\xi+\eta}
$$

where $\eta=k_{1} x+L_{1} y-\omega_{1} t+\gamma_{1}$ and $\xi=k_{2} x+L_{2} y-\omega_{2} t+\gamma_{2}$. Theoretically, we can obtain the double solitary wave solution of KP equation (1).

In this case, we introduce an extended double-soliton test function:

$$
F(x, y, t)=1+e^{\eta}+G(\xi)+A G(\xi) e^{\eta},
$$

where $\eta=k_{1} x+L_{1} y-\omega_{1} t+\gamma_{1}$ and $\xi=k_{2} x+L_{2} y-\omega_{2} t+\gamma_{2}$. The unknown real function $G(\eta)$ satisfies the following auxiliary equation:

$$
\left(G^{\prime}(\xi)\right)^{2}=h_{0}+h_{1} G(\xi)+h_{2} G^{2}(\xi)
$$

The parameters $k_{1}, k_{2}, L_{1}, L_{2}, \omega_{1}$, and $\omega_{2}$ are nonzero constants to be determined, and $\gamma_{1}$, and $\gamma_{2}$ are phase constants. $A$ is a real number that stands for the resonant factor of traveling wave.

Substituting (18) and (19) into (3) and collecting the coefficients of $e^{\eta}, G(\xi)$, and $G^{\prime}(\xi)$, one yields a nonlinear algebraic system of parameters $h_{0}, h_{1}, h_{2}, k_{1}, k_{2}, L_{1}, L_{2}, \omega_{1}$, and $\omega_{2}$.

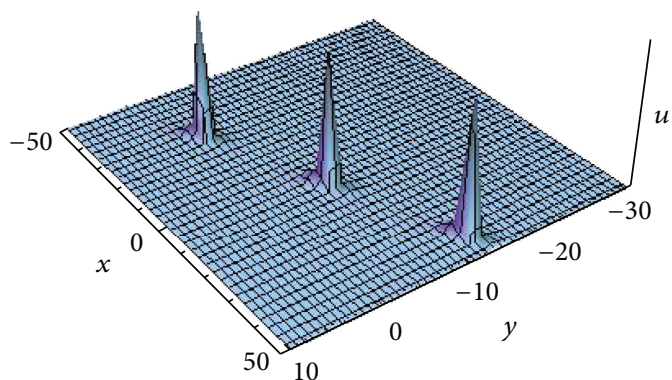

FIGURE 3: A snapshot of irregular cusped solitary wave expressed by (13) at $t=0.85$.

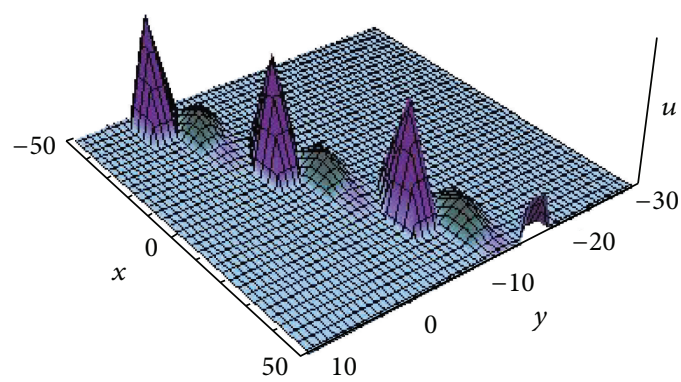

FIGURE 4: A snapshot of irregular cusped solitary wave expressed by (13) at $t=1.1$.

In particular, $h_{0}=-q r, h_{1}=q+r$, and $h_{2}=-1$ are taken to solve the nonlinear algebraic system whose $q$ and $r$ are undetermined constants; we obtain

$$
\begin{gathered}
q=-2-r, \\
L_{1}=\frac{-\sqrt{3 p^{2}} k_{2}^{2}\left(k_{1}^{2}+k_{2}^{2}\right)+p^{2} k_{1} k_{2} L_{2}}{p^{2} k_{2}^{2}}, \\
A=0, \\
\omega_{1}=\frac{-k_{1} k_{2}^{3}-4 k_{1}^{3} k_{2}^{3}+2 \sqrt{3 p^{2}} k_{2}^{2}\left(k_{1}^{2}+k_{2}^{2}\right) L_{2}-p^{2} k_{1} k_{2} L_{2}^{2}}{k_{2}^{3}}, \\
\omega_{2}=\frac{-k_{2}^{2}+4 k_{2}^{4}-p^{2} L_{2}^{2}}{k_{2}} .
\end{gathered}
$$

For (19), when $h_{0}=-q r, h_{1}=q+r$, and $h_{2}=-1$, it allows the fundamental solution as follows:

$$
G(\xi)=\frac{1}{2}(q+r-(q-r) \cos (\xi))
$$

According to the expressions of $\omega_{1}$, and $L_{1}$ in (20) and the significance of coefficient $p$ for KP equation, it is necessary 


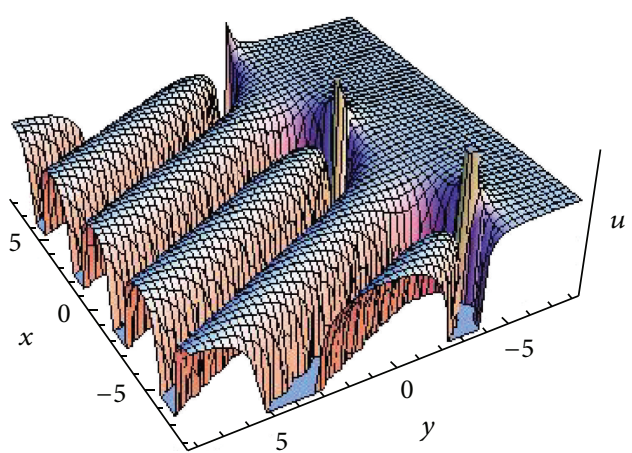

FIGURE 5: A snapshot for assimilation of solitary wave and periodic traveling wave expressed by (22) at $t=3$, where $x \in[-8,8]$ and $y \in[-8,8]$.

that $p^{2}=1$. Substituting (20) and (21) into (18), by (2), we obtain an exact traveling wave solution for KP-I equation:

$$
\begin{gathered}
u(x, y, t)=2(1+r)\left(k_{1}^{2} e^{\eta} \cos (\xi)+2 k_{1} k_{2} e^{\eta} \sin (\xi)\right. \\
\left.-k_{2}^{2}\left(1+r+e^{\eta} \cos (\xi)\right)\right) \\
\times\left(\left(e^{\eta}+(1+r) \cos (\xi)\right)^{2}\right)^{-1},
\end{gathered}
$$

where $\eta=k_{1} x+L_{1} y-\omega_{1} t+\gamma_{1}$ and $\xi=k_{2} x+L_{2} y-\omega_{2} t+$ $\gamma_{2}, k_{1}, k_{2}, L_{2}$, and $r$ are arbitrary nonzero real constants, and $\omega_{1}, \omega_{2}$, and $L_{1}$ satisfy

$$
\begin{gathered}
\omega_{1}=\frac{2 \sqrt{3} k_{2}\left(k_{1}^{2}+k_{2}^{2}\right) L_{2}-k_{1} k_{2}^{2}-4 k_{1}^{3} k_{2}^{2}-k_{1} L_{2}^{2}}{k_{2}^{2}}, \\
\omega_{2}=\frac{4 k_{2}^{4}-k_{2}^{2}-L_{2}^{2}}{k_{2}} \\
L_{1}=-\frac{\sqrt{3} k_{2}\left(k_{1}^{2}+k_{2}^{2}\right)-k_{1} L_{2}}{k_{2}} .
\end{gathered}
$$

The solution expressed by (22) is a new type of traveling wave solution for KP-I equation. Choosing a set of parameters

$$
\begin{array}{ccc}
k_{1}=0.2, & k_{2}=0.8, & L_{2}=0.4, \\
\gamma_{1}=0, & \gamma_{2}=0, & r=1,
\end{array}
$$

the assimilation of solitary wave and periodic traveling wave is exhibited by Figures 5 and 6 .

\section{Conclusions}

In this paper, we consider the joint application of bilinear operator and F-expansion method. Choosing the KP equation as an example, we obtain a new type of solitary wave solution which possesses cusped structure by the singlesoliton test approach. The regular and irregular parametric relationships of cusped solitary wave solution are discussed; an interesting phenomenon is found where irregular cusped

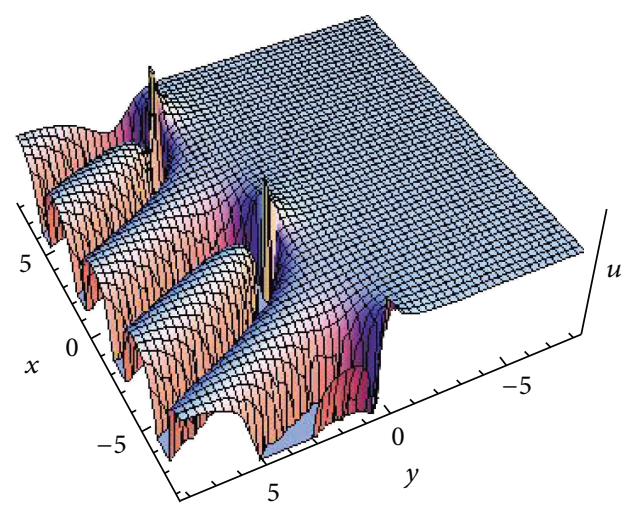

FIGURE 6: A snapshot for assimilation of solitary wave and periodic traveling wave expressed by (22) at $t=-3$.

solitary wave periodically blows up in finite time. Furthermore, an extended double-soliton test method is applied to obtain a new type of exact traveling wave solution of KP-I equation. By numerical simulation of waveform, a nonlinear phenomenon describing the dynamical behavior of assimilation for solitary wave and periodic traveling wave is found. To our knowledge, it has not yet been found until now. The above results obtained in this paper validate the effectiveness of joint application of bilinear operator and Fexpansion method.

\section{Conflict of Interests}

The authors declare that there is no conflict of interests regarding the publication of this paper.

\section{Acknowledgments}

The work described in this paper was fully supported by NSFC (11161020) and Yunnan Educational Science Foundation (08Y0336).

\section{References}

[1] M. L. Wang, "Solitary wave solutions for variant Boussinesq equations," Physics Letters A, vol. 199, no. 3-4, pp. 169-172, 1995.

[2] A. Ebaid, "Exact solitary wave solutions for some nonlinear evolution equations via Exp-function method," Physics Letters A, vol. 365, no. 3, pp. 213-219, 2007.

[3] M. Song, X. Hou, and J. Cao, "Solitary wave solutions and kink wave solutions for a generalized KDV-mKDV equation," Applied Mathematics and Computation, vol. 217, no. 12, pp. 5942-5948, 2011.

[4] Y. Zhou, M. Wang, and T. Miao, “The periodic wave solutions and solitary wave solutions for a class of nonlinear partial differential equations," Physics Letters A, vol. 323, no. 1-2, pp. 77$88,2004$.

[5] M. A. Abdou, "Exact periodic wave solutions to some nonlinear evolution equations," International Journal of Nonlinear Science, vol. 6, no. 2, pp. 145-153, 2008. 
[6] Z. Dai, J. Liu, X. Zeng, and Z. Liu, "Periodic kink-wave and kinky periodic-wave solutions for the Jimbo-Miwa equation," Physics Letters. A, vol. 372, no. 38, pp. 5984-5986, 2008.

[7] J. Li and Y. Zhang, "Exact loop solutions, cusp solutions, solitary wave solutions and periodic wave solutions for the special $\mathrm{CH}$ DP equation," Nonlinear Analysis: Real World Applications, vol. 10, no. 4, pp. 2502-2507, 2009.

[8] M. A. Abdou and A. A. Soliman, "Variational iteration method for solving BURger's and coupled BURger's equations," Journal of Computational and Applied Mathematics, vol. 181, no. 2, pp. 245-251, 2005.

[9] E. M. Zayed, T. A. Nofal, and K. A. Gepreel, "On using the homotopy perturbation method for finding the travelling wave solutions of generalized nonlinear Hirota-Satsuma coupled KdV equations," International Journal of Nonlinear Science, vol. 7, no. 2, pp. 159-166, 2009.

[10] M. Madani, M. Fathizadeh, Y. Khan, and A. Yildirim, "On the coupling of the homotopy perturbation method and Laplace transformation," Mathematical and Computer Modelling, vol. 53, no. 9-10, pp. 1937-1945, 2011.

[11] D. Feng and K. Li, "Exact traveling wave solutions for a generalized Hirota-Satsuma coupled KdV equation by Fan subequation method," Physics Letters A, vol. 375, no. 23, pp. 22012210, 2011.

[12] Y. Xie, S. Tang, and D. Feng, "New exact solutions to the generalized KdV equation with generalized evolution," Pramana, vol. 78, no. 4, pp. 499-511, 2012.

[13] A. Bekir and E. Aksoy, "Exact solutions of nonlinear evolution equations with variable coefficients using exp-function method," Applied Mathematics and Computation, vol. 217, no. 1, pp. 430-436, 2010.

[14] Z. Dai, S. Li, Q. Dai, and J. Huang, "Singular periodic soliton solutions and resonance for the Kadomtsev-Petviashvili equation," Chaos, Solitons \& Fractals, vol. 34, no. 4, pp. 1148-1153, 2007.

[15] Z.-D. Dai, S.-L. Li, D.-L. Li, and A.-J. Zhu, "Periodic bifurcation and soliton deflexion for kadomtsev-petviashvili equation," Chinese Physics Letters, vol. 24, no. 6, pp. 1429-1432, 2007.

[16] M. Wang, X. Li, and J. Zhang, “The $G^{\prime} / G$-expansion method and travelling wave solutions of nonlinear evolution equations in mathematical physics," Physics Letters A, vol. 372, no. 4, pp. 417-423, 2008.

[17] R. Abazari, "Application of $\left(G^{\prime} / G\right)$-expansion method to travelling wave solutions of three nonlinear evolution equation," Computers and Fluids, vol. 39, no. 10, pp. 1957-1963, 2010.

[18] M. A. Abdou, "The extended F-expansion method and its application for a class of nonlinear evolution equations," Chaos, Solitons \& Fractals, vol. 31, no. 1, pp. 95-104, 2007.

[19] Y. He, Y.-M. Zhao, and Y. Long, "New exact solutions for a higher-order wave equation of KdV type using extended Fexpansion method," Mathematical Problems in Engineering, vol. 2013, Article ID 128970, 8 pages, 2013.

[20] A. Ebaid and E. H. Aly, "Exact solutions for the transformed reduced Ostrovsky equation via the $F$-expansion method in terms of Weierstrass-elliptic and Jacobian-elliptic functions," Wave Motion, vol. 49, no. 2, pp. 296-308, 2012.

[21] H. Kumar and F. Chand, "Applications of extended F-expansion and projective Ricatti equation methods to $(2+1)$-dimensional soliton equations," AIP Advances, vol. 3, no. 3, Article ID 032128, 2013.
[22] D.-Q. Xian and H.-L. Chen, "Symmetry reductions and new exact non-traveling wave solutions of potential KadomtsevPetviashvili equation with p-power," Applied Mathematics and Computation, vol. 216, no. 1, pp. 70-79, 2010.

[23] S. V. Manakov and P. M. Santini, "Wave breaking in solutions of the dispersionless Kadomtsev-Petviashvili equation at at finite time," Theoretical and Mathematical Physics, vol. 172, no. 2, pp. 1118-1126, 2012.

[24] C. Klein and J.-C. Saut, "Numerical study of blow up and stability of solutions of generalized Kadomtsev-Petviashvili equations," Journal of Nonlinear Science, vol. 22, no. 5, pp. 763811, 2012. 


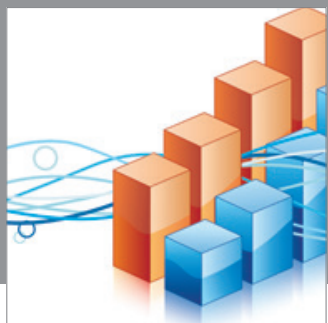

Advances in

Operations Research

mansans

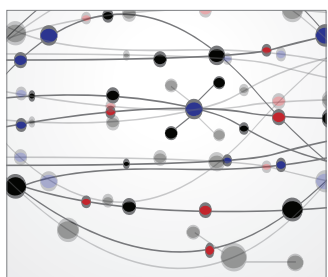

The Scientific World Journal
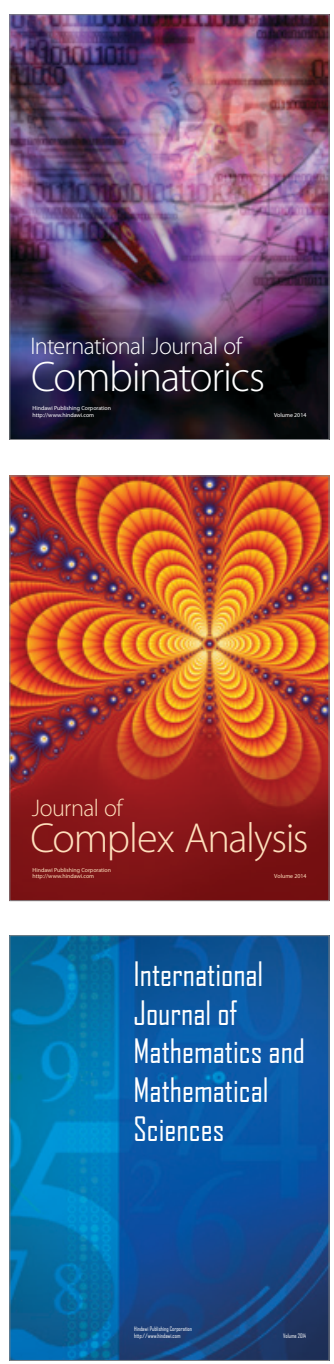
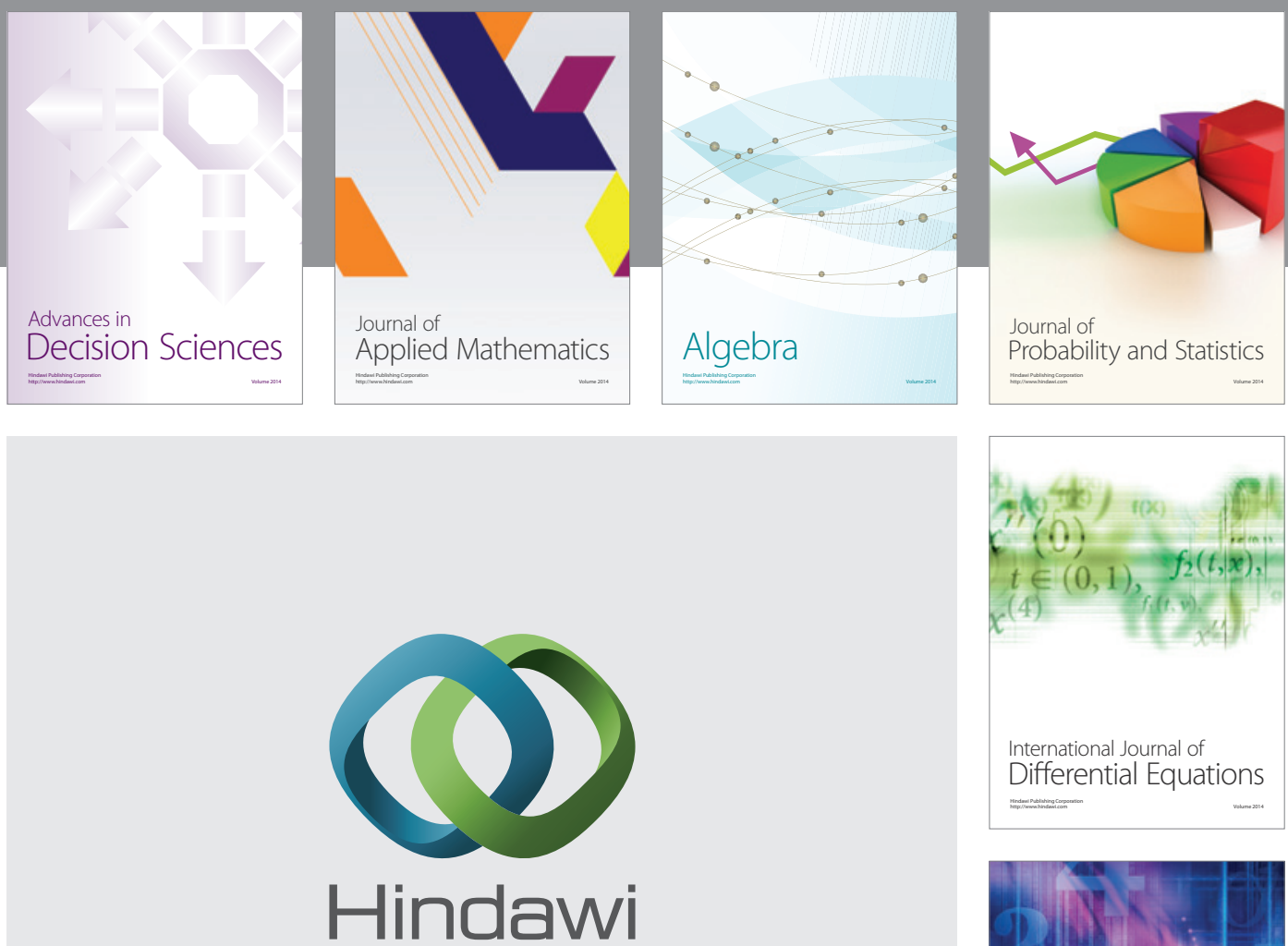

Submit your manuscripts at http://www.hindawi.com
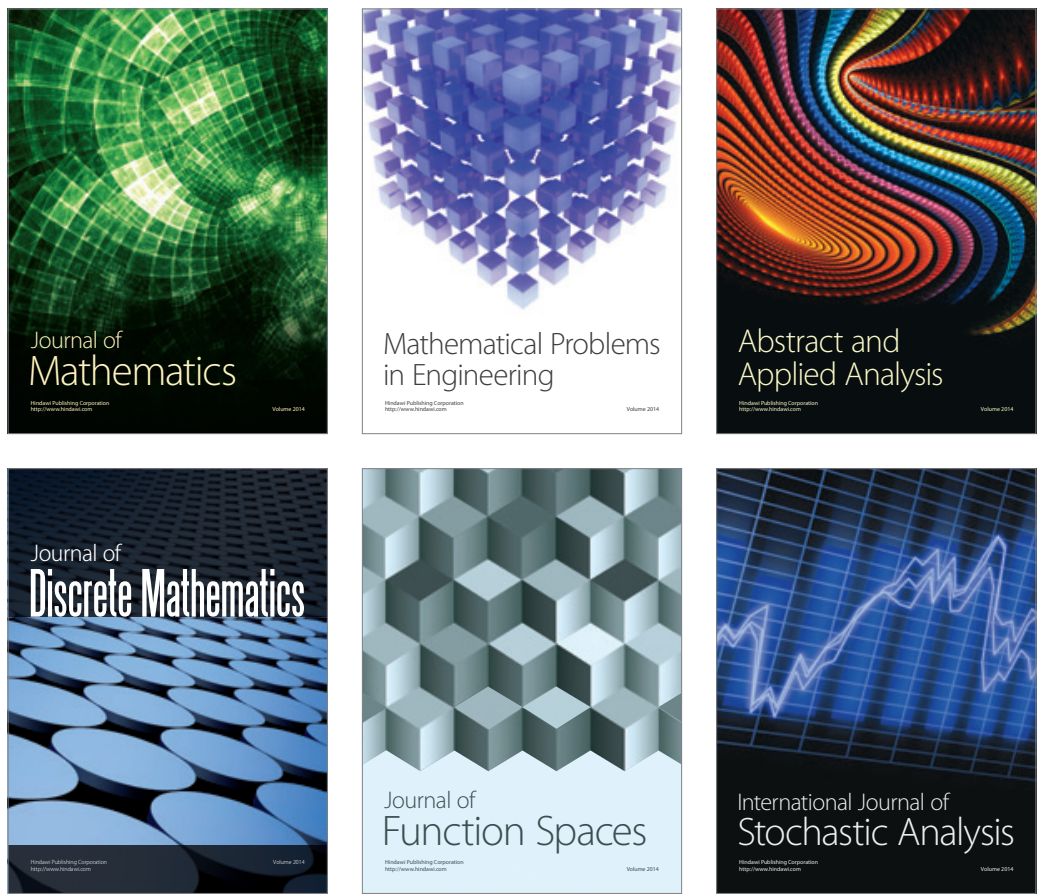

Journal of

Function Spaces

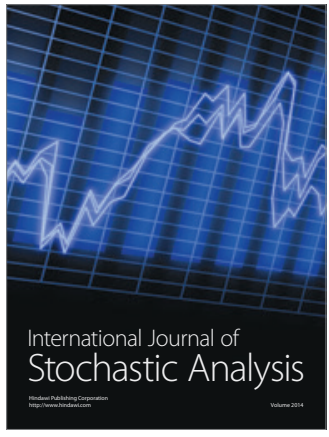

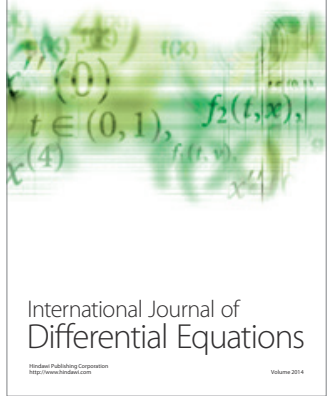
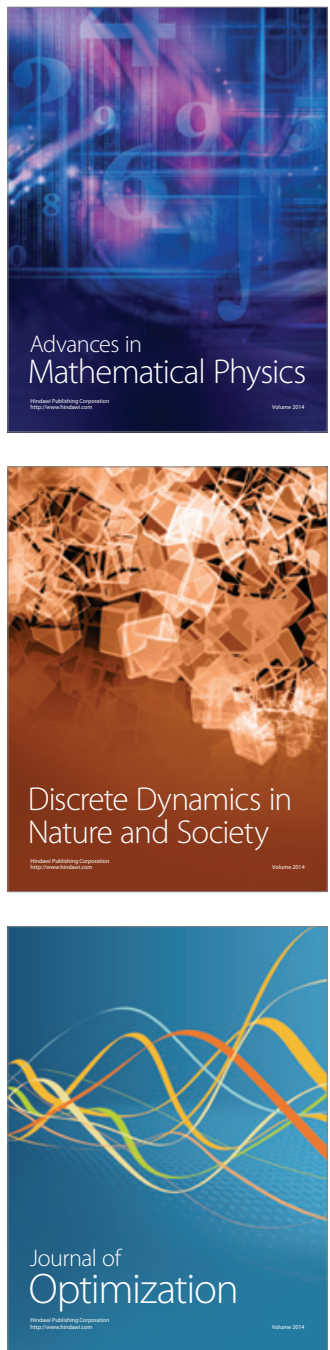